\title{
A Performance Comparison of Reliable-AODV for MANET in NS-2, NS-3 and Qualnet
}

\author{
Mazher Sarfaraz Khan \\ Assistant Professor \\ Marathwada Institute of Technology, Aurangabad
}

\author{
Sayyad Ajij D, PhD \\ Assistant Professor \\ Marathwada Institute of Technology, Aurangabad
}

\begin{abstract}
Ad hoc on demand distance vector is most suited for Mobile ad hoc network under random topology. MANET provide a backbone to recent wireless communication technologies. MANET in which nodes communicate with other nodes without any central support. Improvement in AODV is done by cross-layer design to avoid link crash. New ideas for crosslayer design has been suggested and implemented but it needs to be tested in the different simulator for its reliability. This paper intends to check the reliability of new ideas for crosslayer design in different simulators such as Qualnet, NS2, and NS3. Each simulator has its own feature, and they work on different platforms. Now in this paper, it will be clear that which simulator is most suitable for MANET simulation. In this paper studies and experimentation shows that NS3 and Qulanet are the best real-time simulator. Peculiarities of each simulator with its simulation settings are discussed in detail.
\end{abstract}

\section{General Terms}

Ad hoc on demand distance vector, Mobile Ad hoc network, Received signal strength, Cross layer design

\section{Keywords}

AODV, MANET, Cross layer Design, NS-2, NS-3, Qualnet

\section{INTRODUCTION}

Modern technologies are impediment without wireless communication. In each industrial automation and machine approach, wireless communication is must be craved. Internet of thing and industry 4.0 are emerging fields of wireless communication. Now, $4 \mathrm{G}$ guided network moving toward $5 \mathrm{G}$ network for more advancement and the requirement of society as a part of wireless communication. MANET is an organ of wireless communication which is also moving in parallel to recent wireless technologies. Now there is a need for reliable network formation using MANET which will full fill the requirement of Military and disaster management. This paper includes different sections, Section-1 is describing in brief about MANET and AODV, Section-II about related literature survey of the simulator, Section -III about proposed crosslayer design, Section-IV explaining each simulator in details, Section-V result, and discussion.

\section{MANET FEATURES}

1) Dynamic typologies

2) Bandwidth constrained variable

3) Energy constrained operation

4) Physical security is limited

5) No infrastructure needed as in Figure 1

6) Easy to design.

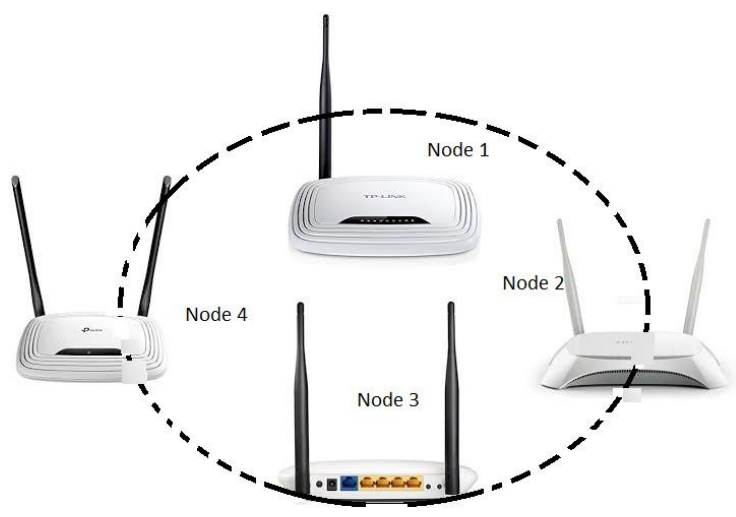

Fig 1: MANET operation

\subsection{Issues in MANET}

1) Packet losses in ad hoc networks

2) Link failures or link breakages

3) Node failures

4) Quality of service

5) Changes in

6) Mobility

7) Security

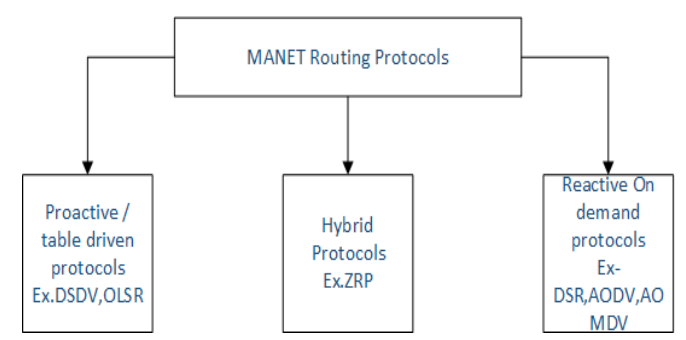

Fig 2: Classification of routing Protocols

\subsection{AODV routing Protocol}

Figure 2 describe types of routing protocol. Most efficient for MANET is AODV under random typologies and mobility.

\section{B. Essential functionality of $A O D V$ includes}

1) RREQ and RREP messages (for route discovery) as in Figure 3

2) RERR messages, HELLO messages, precursor lists (for route maintenance) as in Figure 3

3) Sequence numbers

4) Hop counts

5) Expanding ring search 


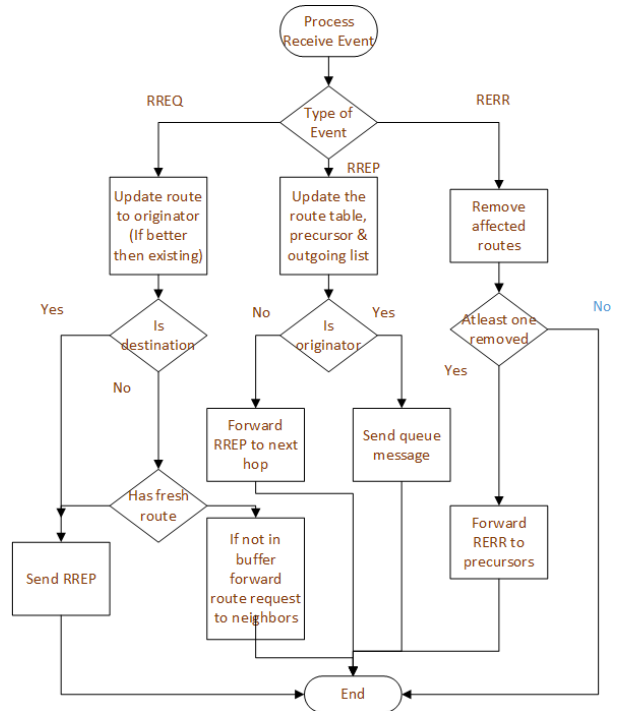

Fig 3 : Event Processing in AODV

Figure 3 describe functioning of AODV for all events processing.

from the bottom. The right and left margins should be $1.9 \mathrm{~cm}$ (.75"). The text should be in two $8.45 \mathrm{~cm}(3.33 ")$ columns with a $.83 \mathrm{~cm}(.33 ")$ gutter.

\section{RELATED WORK}

\subsection{Literature survey for Reliable-AODV}

In [1] A. Sarfaraz Ahmed et al. proposed cross-layer design technique for power control in MANETs., it is determined improper to deal with receiving signal strength (RSS)-related issues, changing the physical layer, the network layer and transport layer. The Cross-Layer design method for Power control (CLPC) will increase the transmission power by equalizing the RSS values and to determine the most effective route among the source and the destination. Link breakage can influence the serious impact in the network performance because the routing overhead of distinct route finding is very high Whereas, in the suggested model (CLPC), a dynamic transmission power control algorithm identifies a link breakage if it happens and determines the fresh route to be refreshed in the routing table.

In [2] Mrs Sunita Nandgave et al. described congestion control protocols. Routing in MANET is exciting due to its mobility feature. The mobile type of nodes and changing topology of Mobile Ad hoc Networks (MANETs) head to route failures and demanding the transmission of control packets. Ad hoc Distance Vector (AODV) is appealing as an efficient on- demand AODV is not robust against topology changes as it handles inadequate links due to long hops proposed by shortest path metric routing protocol because of low routing overhead and great achievement. Three approaches are proposed for congestion control. In Reliable AODV signal strength is considered as cross-layer interaction parameter. Received signal strength is received from the physical layer and is checked at the MAC layer whether it is above a specified threshold. If it is over the threshold, then the connection is the powerful, otherwise weak link, which can cause route failure. When a route is found to get fail due to the uncertain signal strength of a node, it will determine another path. Cross-Layer Stability depends on routing mechanism is (CLS_AODV). Where collected signal strength can be utilized to make recognized the link state information for uncertain zone prediction and route state monitoring.
In [3] R. Senthil Kumar et al. introduced a new protocol for the cross-layer scheme to reduce link break in MANETs. This circumstance causes frequent path failure and route reestablishment. Making QoS (Quality of Service) of connections depending on the state of networks. A number of MANET routing protocol becomes efficient and correct. The proposed algorithm is used and implemented based on the prediction of RSS value to improve the performance of the network and reduce the packet re transmission in MANET. Since considered the factor on the prediction of RSS, it results in minimization of packet loss.

In [4] G. Bhoge et al. stated the objective of the paper is Creating a protocol for the network layer and transport layer that save energy, the lifetime of network and congestion control improves, proposed solution for the discussed problem is cross-layer approach combining the network and transport layer.

Mamata Rath et al. in [5] target highly robust and dynamic military work stations, devices and smaller sub-networks in the area of the battlefield, this paper focuses on a powerefficient network layer routing protocol in the network for specifically military application is designed, It uses the power delay optimized AODV protocol,

Muhammad Kamrul Islam et al. [6] explain how to increase various performance parameters, different cross-layering methods are utilized where multiple, Advantages are the simulation of proposed SNR based AODV shows the performance improvement of the proposed SNR-based AODV protocol.

Anshu Chaturvedi et al. [7] explain the aim of the paper is to optimize total utilization of node energy along with route establishment. The proposed solution for the discussed problem when nodes do not have sufficient energy to transmit or receive packets the performance of the network is affected finding these, advantages are less link failure, higher throughput, fewer data dropped and above data delivery rate and smaller delay time for sending data packets,

M. Anand1 et al., [8] objective of the paper is the battery power is an important field in MANET to provide reliable communication without any power failure. Calculate and deter- mine the distance using the RSSI (Received Signal Strength Indication): advantages are IRAODV protocol was implemented to improve the battery power in MANET.

\subsection{Literature survey of simulator}

Selecting a suitable network simulator is a significant task for researchers. There are researchers who have been experimenting with different routing protocols [9] in different simulators with different network parameters [10] to assess the precise performance of network protocols. The design of ns-2 and TOSSIM are analyzed in [11]. In [12] and [13], the authors present a performance comparison of various network simulators such as JavaSim, ns-2, and SSFNET. [14] mainly centers on simulators that are designed for sensor networks. [15] shows a qualitative comparison of ns-2 and OPNET. Moreover, [16] presents a performance comparison of network simulators that are specifically designed for VANETs.

The foremost difference between this paper and earlier published papers is that we compare the most widespread, open source and license simulators. In extension, in this, used a MANET routing protocol, i.e. AODV, to estimate the performance of the network simulators. [17] 
1) MANET Simulation Tools: This section presents the selected network simulators, i.e. ns-2, ns-3 and Qualnet in detail. These simulators are chosen because of their high demanding the research society and maximum researchers utilize them to check their new theories.

a) ns-2: Network Simulator-2 (ns-2) [18] is an open source, discrete event wired and wireless network simulator. It is utilized for the simulation of network protocols with various network typologies. It is proficient of simulating wired and wireless networks. NS-2 was developed in $\mathrm{C}++$ and shows the simulation interface by OTcl, an object-oriented dialect of Tcl. The user describes a network topology by writing OTcl scripts, and then the main NS program simulates that topology with particularized parameters. In ns-2, network animator (NAM) is practiced for the graphical view of the network. ns2 is the most popular and extensively utilized network simulator for research activity. NAM interface includes control features that permit users to forward, pause, stop and play the simulation [17]. The interface of ns-2 is shown in

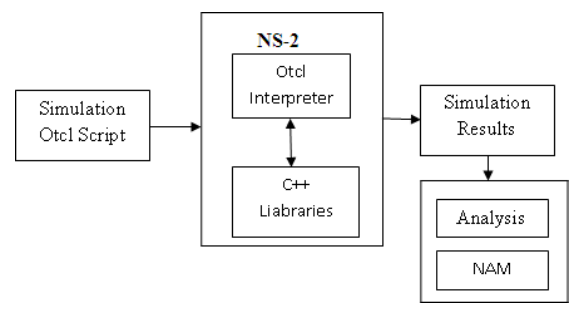

Fig. 4. NS-2 basic architecture

Figure 4 shows basic structure of NS-2 working

Figure 5 demonstrate Protocol stack of NS-2 with reference to OSI modelFigure 6 Shows Nam windows of simulator NS-2

In ns-2, temporary network typologies can be described that are made of routers, links and participated media [19]. The physical activities of the network are treated and queued in

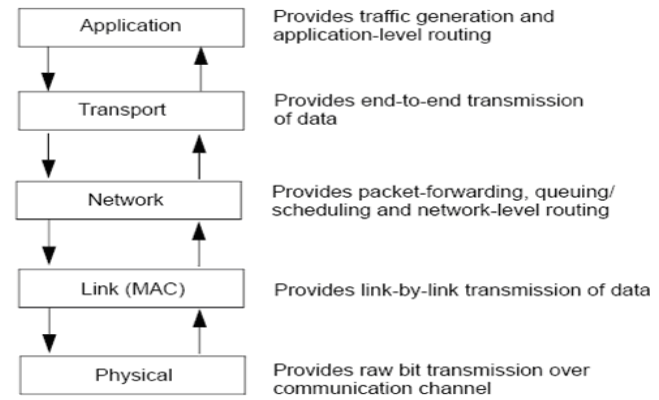

Fig. 5. NS-2 protocol stack

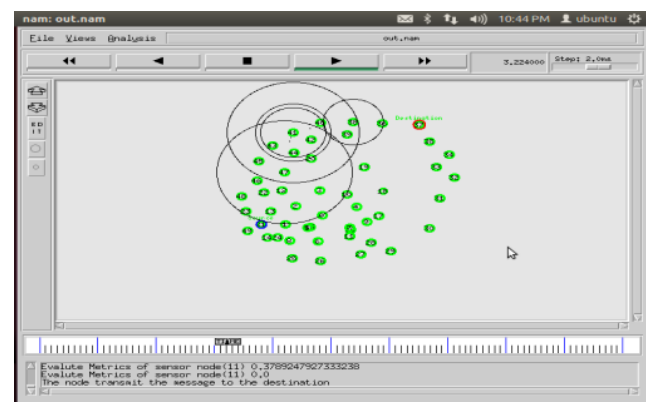

Fig. 6. Nam windows of NS-2

the form of events, in a scheduled order. These events are then treated as per scheduled time that stretches along with the processing of events. However, the simulation is not real time; it is supposed virtual [20] [17]

b) ns-3: The ns-3 project [21] was begun in mid-2006 and is still below huge development. Like ns-2, ns-3 is also an open source, discrete-event network simulator. ns-3 is identified as a replacement of ns-2, not an expansion [22]. As compared to ns-2, it does not possess an OTcl API. It is drafted in C++ language and python. The newest version of ns-3 is ns-3.10 that encourages parallel simulation and has a magnified feature set. In addition, ns-3 network simulations can be executed in $\mathrm{C}++$ and Python [12]. ns-3 interface is shown

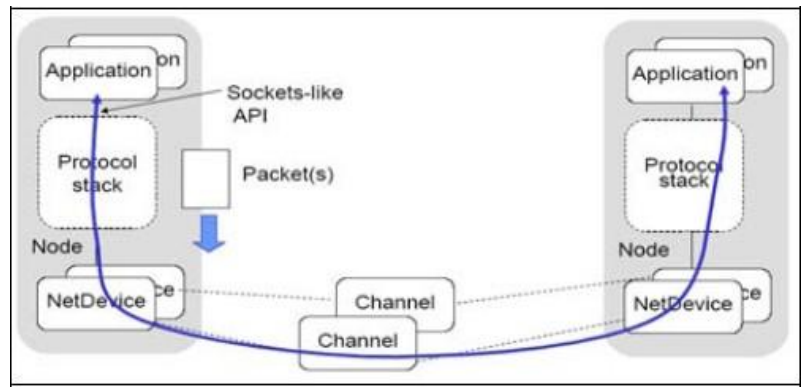

Fig. 7. NS-3 basic architecture [23]

Figure 7 indicate basic architecture and functioning module of NS-3

Figure 10 state Network animator windows of NS-3. ns-3 carries both simulation and emulation using sock- ets. [24] [17].

c) OMNET++: OMNET++ [25] has been accessible to the public since September 1997 and recently has a many numbers of users. As compared to ns-2 and ns-3, OMNET++ is not only created for network simulations. It can be utilized for modeling of multiprocessors, distributed hardware systems and performance calculation of difficult software systems.

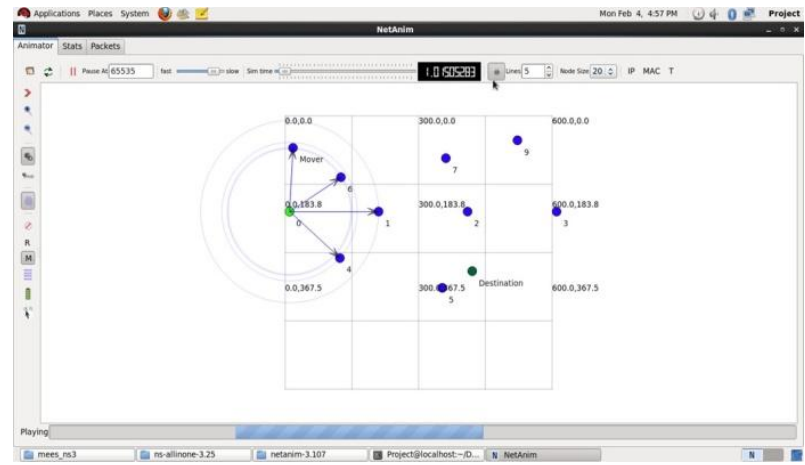

Fig. 8. NetAnim

However, it is mostly used for computer based net- works simulation. OMNET++ is a discrete event, component- based (modular) open architecture simulation framework. [26]. OMNET++ distributions are prepared for both UNIX and Windows systems. It was made utilizing the componentoriented method that supports structured and reusable models. In extension, OMNET++ has a great graphical user interface (GUI) and intelligence support [27] [17]

d) GloMoSiM: Global Mobile Information System Simulator (GloMoSiM) is a simulation environment used for wide-scale wireless networks. GloMoSiM handles parallel discrete-event simulation based on Parsec [28]. In extension, GloMoSiM uses the Parsec compiler to compile the simulation of protocols [29]. 
GloMoSiM is proficient of simulating a network that contains thousands of nodes and various communication links, for example, multi cast and asymmetric links. In addition, GloMoSiM encourages satellite communication, multi-hop wire- less communication, and many of the conventional Internet protocols. GloMoSiM is a library-based sequential and parallel simulator that is produced purely for wireless networks [29]. GloMoSiM has a scalable simulation library that depends on the Parsec simulation environment [30] [31] [17].

e) QualNet: QualNet is a monetary ad hoc network simulator based on the GloMoSim

core. It continues the GloMoSim offer by bringing support, decent documentation, a complete set of user-friendly tools for creating scenarios and analyzing simulation output. QualNet also largely continues the set of models and protocols sup- ported by the original GloMoSim distribution. As it is built on the head of GloMoSim, QualNet is written in Parsec. [32]

Figure 9 is QualNet functional diagram

Figure 10 demonstration of example in QualNet

$\mathrm{C}++$ : For executing new protocols, Qualnet uses $\mathrm{C} / \mathrm{C}++$ and follows a procedural model. Uses the parallel simulation

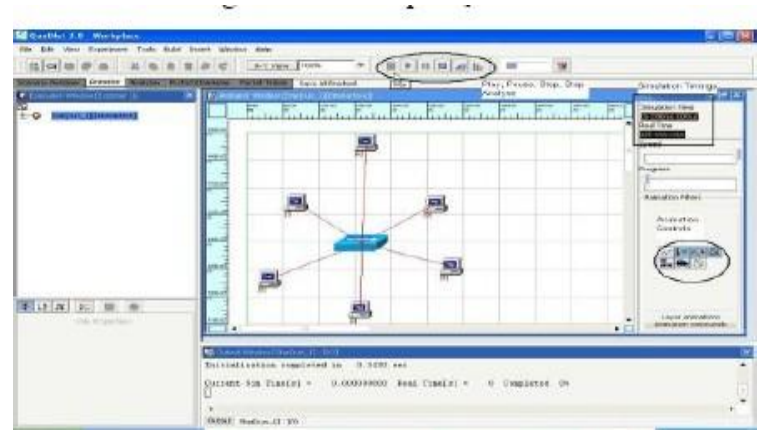

Fig. 9. QualNet Architecture [32]

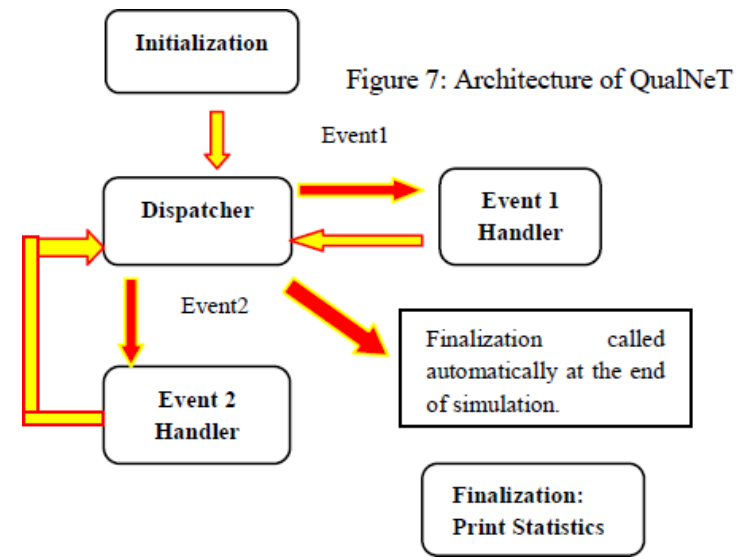

Fig. 10. QualNet example

environment for complex systems (PARSEC) for basic operations hence can run on distributed machines [15]. The component diagram

of QUALNET is given in Figure 9.

2) Selection of Simulators: MANETs simulators show different features and models. The choice of the simulator should be directed by the requirements. Determining the level of de- tails needed is key. If high-precision PHY layers are must, then ns-2 (coupled with the highly-accurate PHY is surely the good choice. If larger scales are required, then parallel simulators are a smart choice. You may also consider highly optimized simulators like ns- 2 coupled with stage simulation. [33]

\section{CROSS LAYER DESIGN IMPLEMENTATION FOR RELIABLE- AODV}

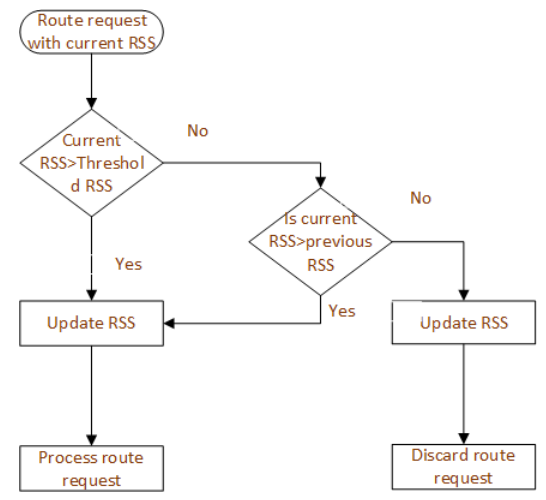

Fig. 11. AODV route request

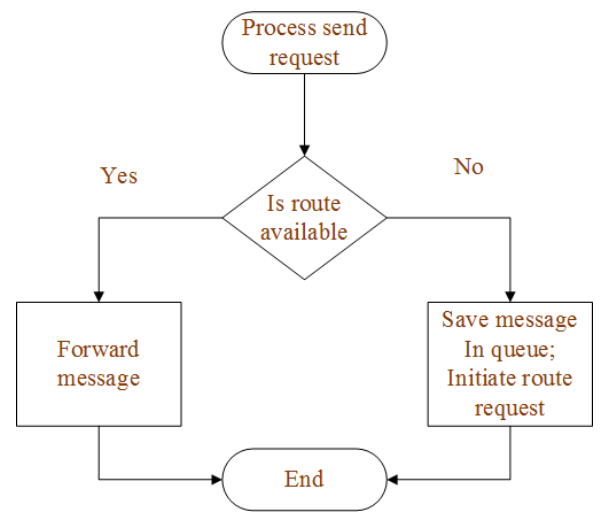

Fig. 12. Cross-layer based route request

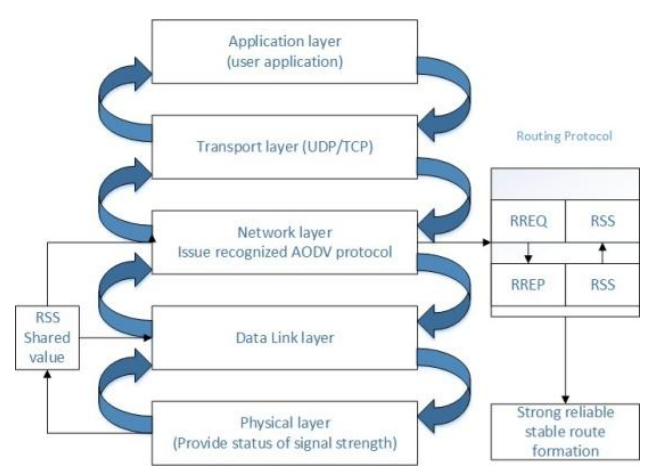

Fig. 13. CLD implementation

Figure 11 shows basic RREQ event handling in AODV which is further modified using RSS value in Figure 12 . Implementation of Reliable -AODV can be understood through Figure 13 which describe how RSS value is taken from physical layer and shared with network layer as crosslayer interaction parameter. So, above approach of ReliableAODV is tested in NS-2, NS-3 and QualNet in succeeding section. 


\section{RESULT AND DISCUSSION}

\section{SIMULATION SCENARIO}

\begin{tabular}{|ll|}
\hline Parameter & CLD-AODV \\
\hline Simulator & NS 2, NS 3, QualNet \\
\hline Speed & 512 bytes \\
\hline Packet Size & 4 packets/s \\
\hline Packet Rate & $1000 * 1000$ \\
\hline Simulation Area & $250 \quad \mathrm{~m}$ Receiver Sensitivity \\
\hline $\begin{array}{l}\text { Max Propagation Range } \\
\text { (Low RSS) } \\
802.11\end{array}$ & CLD-AODE dBm AODV Protocol \\
\hline Routing Protocol & CBR \\
\hline Traffic Type & $10 \mathrm{~s}$ \\
\hline Simulation Time & Two Ray Model Mobility \\
\hline Channel Propagation Model & \\
\hline Random & UDP \\
\hline Transport Layer Protocol & Omni antenna \\
\hline Antenna & $50-200$ \\
\hline Nodes & $10 \mathrm{~J}$ \\
\hline Initial energy & \\
\hline
\end{tabular}

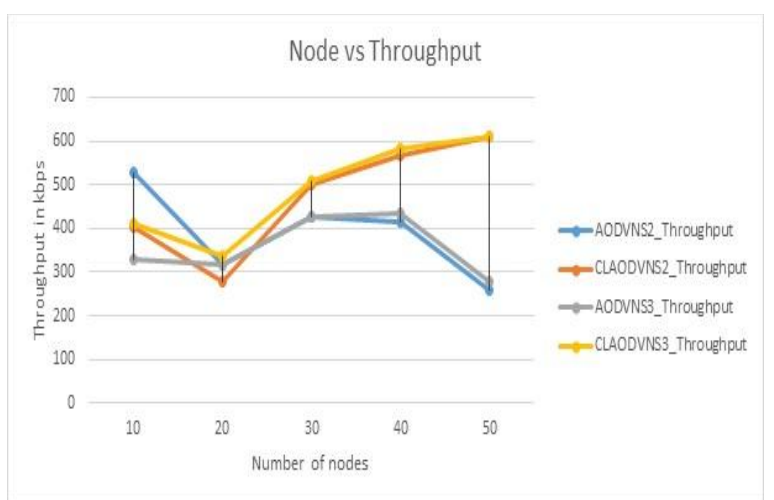

Fig. 14. Node vs Throughput

Result comparison in ns-2 and ns-3 for CLD-AODV (Reliable-AODV) as compared to AODV

Figure 14 shows improved throughput result in both ns- 2 and ns-3 for Reliable-AODV

Figure 15 show throughput variation with respect to speed and in this graph also Reliable-AODV has improved result in ns-2 and ns-3.ns-2 and ns-3 results are at most same here and overlapping.

Figure 16 fading factor is affecting the performance of network but in Reliable-AODV it shows improved result. ns-2 and ns-3 results are overlapping each other

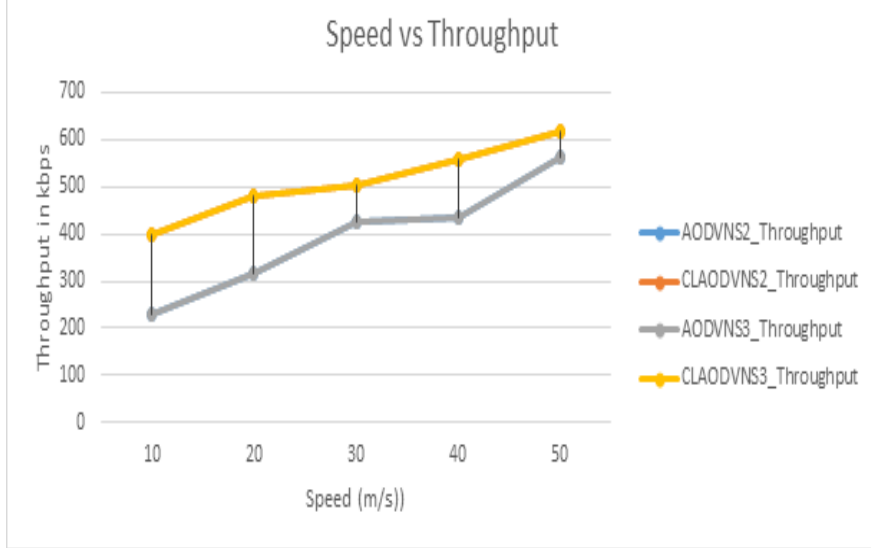

Fig. 15. Speed vs Throughput

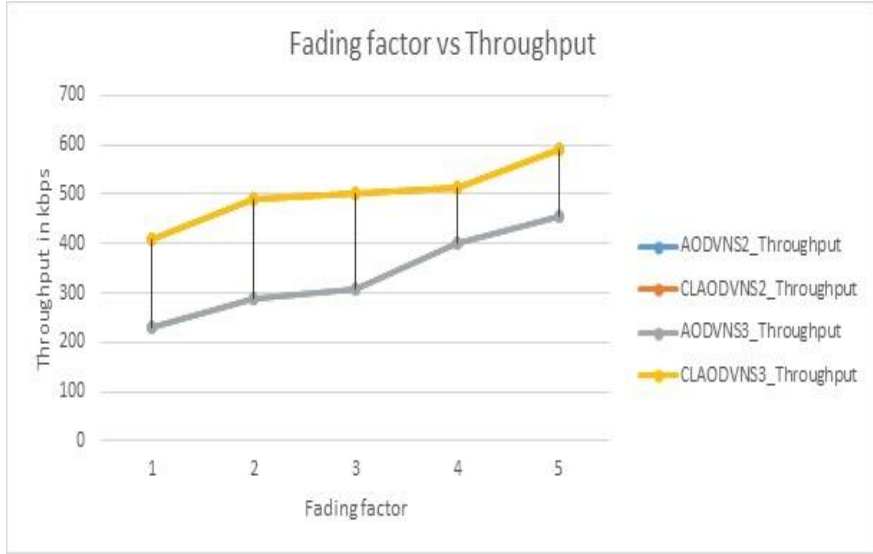

Fig .16. fading factor vs Throughput

CLD-AODV (Reliable-AODV) as compared to AODV in QualNet

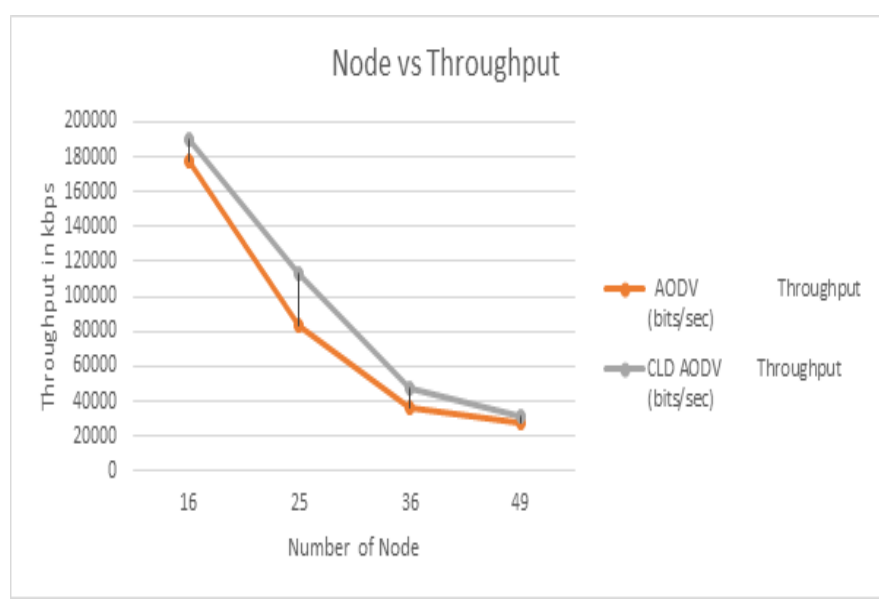

Fig. 17. Node vs Throughput 


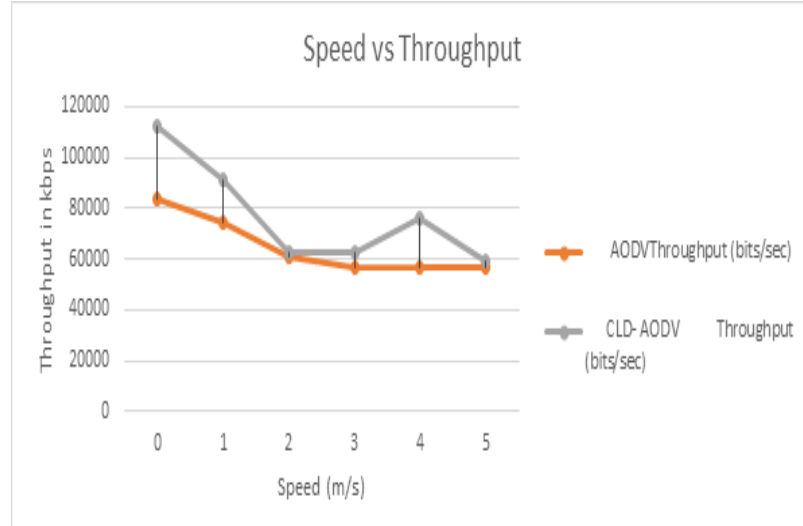

Fig. 18. Speed vs Throughput

Figure 17 shows better results for Reliable-AODV but decrease in throughput as number of nodes increases.

Figure 18 Shows slowly increase in speed causes decreasing performance but Reliable-AODV has increase performance as compared to AODV.

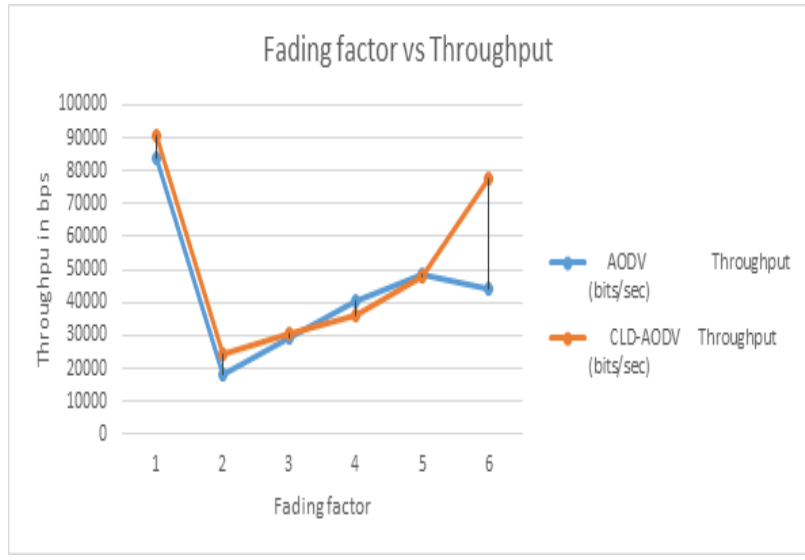

Fig. 19. Fading factor vs Throughput

Figure 19 shows performance under changing fading factor and metric for comparison is throughput.

\section{CONCLUSION}

MANET can be made more stable under versatile conditions my resolving its issue related with link quality. AODV converted into Reliable-AODV by cross layer development using RSS values. From result, it is observed that proposed system gives a better result under different conditions of network. ns-2 and ns-3 result are nearly same but Qualnet gives result with accuracy. In open source ns-3 is the best simulator and otherwise Qualnet performance is too much accurate. Reliable- AODV can be used in MANET for military application and disaster management as it shows great improvement in the performance.

\section{REFERENCES}

[1] A. S. Ahmed, T. S. Kumaran, S. A. Syed, and S. S. Cross, "layer design approach for power control in mobile ad hoc networks," Egypt. Informatics J, vol. 1, no. $1-7,2015$.

[2] S. Nandgave-Usturge, "Study of congestion control using AODV and signal strength by avoiding link failure in MANET," 2012

[3] R. P. Kamalakkannan, "A review and design study of cross layer scheme based algorithm to reduce the link break in MANETs," 2013.

[4] G. Bhoge, M. D. Chawhan, and Y. S. V. K, Taksande,"Cross-layer approach for energy \& communication efficient protocol of mobile ad hoc networks", (C . IEEE, 2017.

[5] [5] M. Rathehta, B. K. Pattanayak, and B. Pati, "Energy Efficient MANET Protocol Using Cross Layer Design for Military Applications," Defence Science Journal, vol. 66, no. 2, 32016.

[6] "Kamrul Islam,Rong Ke Liu,"Cross-Layer Optimization of AODV Rout- ing Protocol For Mobile Ad-Hoc Network (MANET)”,Published by Atlantis Press,” 2013.

[7] D. N. Goswami, Chaturvedi and Shivjay. Singh,"Energy Aware Route Selection Algorithm for Cross Layer Design over MANET",978-1-4673- 7231-2/15/\$31.00 @ IEEE, 2015.

[8] "Anand1 T. Sasikala2, "Efficient energy optimization in mobile ad hoc network (MANET) using."

[9] S. R. Das, C. E. Perkins, and E. M. Royer, "Performance comparison of two on-demand routing protocols for ad hoc networks," 2000.

[10] A. R. Khan, S. A. Madani, K. Hayat, and S. U. Khan, "Clustering- based power-controlled routing for mobile wireless sensor networks," International Journal of Communication Systems, vol. 25, no. 4, pp. 529-542, 2012.

[11] M. Karl, A Comparison of the architecture of network simulators NS-2 and TOSSIM, 12005.

[12] E. Weingarten, H. V. Lehn, and K. Wehrle, A performance comparison of recent network simulators, 2009.

[13] D. M. Nicol, "Comparison of Network Simulators Revisited," 2002. [Online]. Available: htttp://www.ssfnet.org/Exchange/gallery/dumbbell/ dumbbellperformance-May02.pdf

[14] H. Sundani, H. Li, V. K. Devabhaktuni, and M. Alam, "Wireless Sensor Network Simulators A Survey and Comparisons," International Journal of Computer Networks, vol. 2, pp. 249-265, 42011.

[15] B. Schilling and J. Hahner, "Qualitative Comparison of Network Simu- lation Tools, Modeling and Simulation of Computer Systems," 2005.

[16] A. Hassan, VANET Simulation, Halmstad: University,May, 2009.

[17] S. M. Bilalb and M. Othmana, "A performance comparison of network simulators for wireless networks," 2013.

[18] "The network simulator. ns-2." [Online]. Available: http://www.isi.edu/ nsnam/ns.

[19] C. H. Vrije and M. Hofmann, Performance Compari-son of Reliable Multicast Protocols using the Network Simulator ns-2, 1998.

[20] P. Yinfei, "Design Routing Protocol Performance Comparision in NS2: AODV Comparing to DSR as Example," 2010.

[21] T. R. Henderson, S. Roy, S. Floyd, and G. F. Riley, ns-3 project goals, New York, USA, 2006. 
[22] G. Carneiro, P. Fortuna, and M. Ricardo, FlowMonitor a network monitoring framework for the Network Simulator 3 (NS-3), 2009.

[23] R. Chaudhary, S. Sethi, R. Keshari, and S. Goel, "A study of comparison of Network Simulator-3 and Network Simulator-2," IJCSIT) Interna- tional Journal of Computer Science and Information Technologies, vol. 3 , no. 1, pp. 3085-3092, 2012.

[24] T. Saeed, H. Gill, Q. Fei, Z. Zhang, and B. T. Loo, An Open-source and Declarative Approach to-wards Teaching Large-scale Networked Systems Programming, Toronto, Canada, 2011.

[25] A. Varga and R. Hornig, "“'An overview of the $\mathrm{OMNeT}++$ simulation environment," Interna-tional Conference on Simulation Tools and Tech- niques for Communications, Networks and Systems," 2008.

[26] A. Varga, "” THE OMNET++ DISCRETE EVENT SIMULATION SYS- TEM" European Simulation Multiconference," 2003.

[27] A. Varga and A. Y. Sekercioglu, “'”Parallel Simulation
Made Easy with OMNeT++" 15th European Simulation Symposium," 2003.

[28] R. Bagrodia and R. Meyerr, "PARSEC: A Parallel Simulation Environ- ment for Complex System," 1997.

[29] "GloMoSiM, http://pcl.cs.ucla.edu/projects/glomosim/, ac-cessed Sept 20th,” 2011.

[30] L. Bajaj, M. Takai, R. Ahuja, K. Tang, R. Bagrodia, and M. Gerla, "GloMoSiM: A Scalable Network Simulation Environment," IEEE Transactions on Reliability - TR, 1999.

[31] X. Zeng, R. Bagrodia, and M. Gerla, GloMoSiM: A Library for parallel simulation of Large scale wireless networks, 1998.

[32] S. Siraj, A. Gupta, and R. Badgujar, "Network simulation tools survey," International Journal of Advanced Research in Computer and Commu- nication Engineering, vol. 1, no. 4, pp. 199-206, 2012.

[33] L. Hogie, P. Bouvry, and F. Guinand, An overview of manets simulation, 2006, vol. 150 , no. 1 . 